\title{
The Total Amount and Composition Determination of Crocetin and Its Derivatives from Gardenia Yellow by HPLC-MS-MS
}

\author{
Bodi Hui $^{1 *}$, Jin Huang ${ }^{1,2}$ \\ ${ }^{1}$ Department of Food Science, Beijing Union University, Beijing 100023, P. R. China \\ ${ }^{2}$ COFCO Nutrition and Health Research Institute Co Ltd, Beijing 102209, P.R. China
}

*Corresponding author: Bodi Hui, Department of Food Science, Beijing Union University, Beijing 100023, P. R. China.

Received date: January 15, 2021 ; Accepted date: April 27, 2021; Published date: May 26,2021

Citation: Bodi Hui, Jin Huang, (2021). The Total Amount and Composition Determination of Crocetin and Its Derivatives from Gardenia Yellow by HPLC-MS-MS. J. Nutrition and Food Processing, 4(3); DOI:10.31579/2637-8914/037

Copyright: (C) 2021 Chen Pan, This is an open access article distributed under the Creative Commons Attribution License, which permits unrestricted use, distribution, and reproduction in any medium, provided the original work is properly cited.

Abstract
Consisting of crocetin derivatives as major color materials, gardenia yellow is a water-soluble food
colorant extracted from the fruits of Gardenia jasminoides Ellis. However, as color materials, the
total crocetin and its derivative amount and composition cannot be determined because the structures
of some crocetin derivatives are currently unknown and the commercially-available reference
sample is still short. This study aims to develop a method for the total amount and composition
determinations of crocetin and its derivatives from gardenia yellow. In practice, the absorption
coefficient of each crocetin derivative is calculated from that of crocetin and the negative correlation
of absorbance with molecular mass of each derivative, while the molecular mass of each crocetin
derivative is substituted by the $\mathrm{m} / \mathrm{z}$ value of its parent ion on MS. The relative standard deviation
(RSD) of reproducibility was $3.17 \%$. The recovery of added external reference sample was
$\geq 97 \pm 1.61 \%$. The minimum detection limit was $7.44 \mu \mathrm{g} / \mathrm{mL}$.
Keywords: gardenia yellow; crocetin; crocetin derivative; crocin; gardenia jasminoides ellis;
high performance liquid chromatography- mass spectrometry/ mass spectrometry (HPLC-MS/MS);
crocetin esters; major fragmentation

\section{Practical Applications}

In the industrial base, a method to determine the absorption coefficient, normally presented as a color value, of gardenia yellow is currently applied for the product quality control scheme. Under such conditions, the product quality was controlled by its color shade but not mass. It is expected that this approach will be replaced by the method developed in this study for the product quality control scheme. By this method, the color value will be substituted by the mass amount of the product for its quality control. This effort will provide a possibility to verify the biological functions and evaluate the safety of gardenia yellow.

\section{Introduction}

Gardenia yellow is a product that is usually used in foods as a colorant under the category of food additive in eastern Asian countries, especially in China, Japan and South Korea (World Health Organization). The product is usually extracted from the fruits of Gardenia jasminoides Ellis by $\mathrm{EtOH}$ with further purification [1-5]. Crocetin and its derivatives are the major color materials of the product [6]. Apart from crocetin, the product usually comprises more than 15 species of crocetin derivatives, mainly esters.
The root structure of the derivatives, crocetin, is a $\mathrm{C}_{20}$ carotenoid with the molecular formula and mass of $\mathrm{C}_{20} \mathrm{H}_{24} \mathrm{O}_{4}$ and $328.40 \mathrm{Da}$. Crocin was the first discovered crocetin ester (from Crocus sativus L.) in nature, and occurs in the fruits of Gardenia jasminoides Ellis with the largest amount. Its molecular structure has been well elucidated. In subsequent studies, Crocus sativus L. was the primary focus in understanding the structure of crocetin esters. In 1975, several carotenoid glycosyl esters were isolated and identified from by ultraviolet-visible spectroscopy (UV/VIS), infrared spectroscopy (IR) , ${ }^{1} \mathrm{H}$ nuclear magnetic resonance $\left({ }^{1} \mathrm{H}-\mathrm{NMR}\right)$ and mass spectrometry (MS) [7]. Sujata V. et al further confirmed that crocetin esters were the metabolites in the followers of Crocus sativus $\mathrm{L}$. A number of crocetin esters and their geometrical isomers were then identified by HPLC-PDA-MS [8-10]. Pfister S. et al first reported the isolation and structure elucidation of crocetin esters from the fruits of Gardenia jasminoides Ellis [11]. The identification and structure elucidation of crocetin esters were then undertaken [14-16]. It is believed that a continuous effort to elucidate the structures of crocetin esters will not stop until the structures of all esters are known from the fruits of gardenia yellow. The molecular structures of crocetin, crocetin ester and crocin, the most common encountered crocetin ester in gardenia yellow are given in Figures 1(a), (b) and (c). 


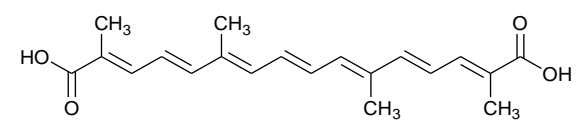

(a)<smiles>CC(/C=C/C=C(\C)C(=O)O)=C\C=C\C=C(/C)C(=O)O</smiles>

(b)

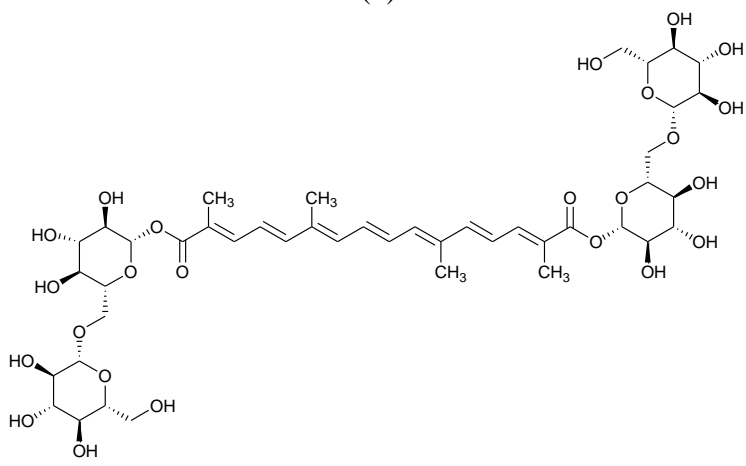

(c)

Figure 1. Molecular structures of crocetin (a), crocetin ester (b) and crocin (c).

Because the structures of some derivatives are still unknown and the commercially available reference samples of all the derivatives, apart from crocetin-di- $\beta$-D-gentiobiosyl ester (crocin), are lacking in the market, the amount and composition of color materials, including crocetin and its derivatives from gardenia yellow cannot be currently determined by either external or internal references. In food industrial base, the addition of gardenia yellow (as a colorant) to food media can only be undertaken by its color value but not quantity. The product quality is usually controlled by its color value but not purity. A number of attempts were made to determine the total amount and composition of crocetin derivatives from gardenia yellow $[17,18]$. Crocin was considered as an external reference sample in those studies. As an alternative, crocetin was also applied as an indicator to evaluate the composition of the crocetin derivatives $[19,20]$.

A method to determine crocetin, its derivative amounts and composition from gardenia yellow was developed by UV-VIS and HPLC-MS-MS in this study based on their electronic absorption spectral characteristics and molecular mass. With this method, the crocetin, its derivative amounts and composition were able to be determined without the aid of external or internal references, even though the molecular structures of some derivatives were unknown.

In industrial base, a method to determine the absorption coefficient, normally presented as color value, of gardenia yellow is currently applied for the product quality control scheme. It is expected that this approach will be replaced by the method developed in this study for the product quality control scheme. By this method, the color value will be substituted by the mass amount of the product for its quality control. This effort will provide a possibility to verify the biological functions and evaluate the safety of gardenia yellow product.

\section{Methods and materials}

\subsection{Materials}

Gardenia yellow product with the label of E500 was extracted from the fruits of Gardenia jasminoides Ellis by EtOH and provided by Henan Zhongda Hengyuan Biotechnology Co. Ltd. EtOH, trifluoroacetic acid (TFA), acetic acid was of AR grade and purchased from a local supplier. Acetonitrile was of HPLC grade and purchased from Dikma Technologies (USA). The water used to prepare the HPLC mobile phase was purified by counter-current osmosis. The crocin reference sample was purchased from Sigma (C/N: 17304-1G). Purity: >95\% (W/W, HPLC).

\subsection{Instrumentation}

UV-VIS spectrophotometer: MultiSpec1500 (Shimadzu); LC-PDA-MSMS: Thermo U-3000-Thermo LTQ Orbitrap XL equipped with PDA, the ion source of ACPI and the central data processing unit of Xcalibur (Thermo Fisher Scientific, USA).

\subsection{Methods}

1.3.1 The specific absorption coefficient determination of gardenia yellow by UV-VIS

This investigation aims to provide the $E_{1 \mathrm{~cm}}^{1 \%}(440 \pm 1 \mathrm{~nm})$ of gardenia yellow. In practice, $0.0502 \mathrm{~g}$ gardenia yellow powders were accurately $(0.0001 \mathrm{~g})$ weighed and then completely dissolved in water to make the total volume of $50 \mathrm{~mL}$. After the solution was diluted 100 times, the absorbance $(A)$ of the solution was measured on a UV-VIS spectrophotometer at $440 \pm 1 \mathrm{~nm}$, with water used as control. The specific absorption coefficient of the product is calculated as follow:

$$
E_{1 \mathrm{~cm}}^{1 \%}(440 \pm 5) n m=\frac{A}{C} \times \frac{1}{100}
$$

Where

$E_{1 \mathrm{~cm}}^{1 \%}(440 \pm 5 \mathrm{~nm})=$ The specific absorption coefficient is defined as the absorbance of the product solution of $1 \%$ concentration, i. e. $1 \mathrm{~g}$ in 100 $\mathrm{mL}$, in a $1 \mathrm{~cm}$ path-length cuvette.

$A=$ Actual absorbance of the product solution

$C=$ Concentration $[\mathrm{g} / \mathrm{mL}]$ of the product solution

1.3.2 The crocetin derivative separation from gardenia yellow by $\mathrm{C} 18$ HPLC

An aliquot of gardenia yellow aqueous solution as described in Section 1.3.1 was filtered by $0.45 \mu \mathrm{m}$ filter before dilution and immediately used for HPLC injection.

The chromatographic profile of gardenia yellow aqueous solution was acquired on C18-HPLC under the following conditions: Diamonsil ${ }^{\mathrm{TM}} \mathrm{C} 18$ 
(4.6 $\times 250 \mathrm{~mm}, 5 \mu \mathrm{m})$ column; Mobile phase $\mathrm{A}=\mathrm{H}_{2} \mathrm{O}-\mathrm{CH}_{3} \mathrm{CN}$-acetic acid at 74.95:25:0.05 (V/V/V); Mobile phase $\mathrm{B}=\mathrm{CH}_{3} \mathrm{CN}$; Linear gradient: Mobile phase $\mathrm{B}$ increased from 0 to $13.1 \%$ (V/V) in 20 minutes subsequently followed by an isocratic elution for 20 minutes; Flow rate = $0.8 \mathrm{~mL} / \mathrm{min}$; Sample injection volume $=20 \mu \mathrm{L}$; Column temperature $=$ $25^{\circ} \mathrm{C}$; PDA monitoring wavelength $=440 \mathrm{~nm}$. The electronic absorption spectrum of each fraction was acquired from 250 to $700 \mathrm{~nm}$ by PDA. The peak area of each fraction was integrated.

\subsubsection{The relative peak area measurement of each fraction}

The relative peak area (\%) of each crocetin derivative fraction is calculated according to formula (2).

$$
\text { Relative peak area } a_{i}=\frac{\text { Peak area }_{i}}{\sum_{i=1}^{n}{\text { Peak } \text { area }_{i}}}
$$

Where

Relative peak area $\mathrm{i}=$ Relative peak area of crocetin derivative $\mathrm{i}$ in total crocetin derivative

Peak area $\mathrm{i}=$ Peak area of crocetin derivative $\mathrm{i}$

$\mathrm{i}=$ Fraction number of crocetin derivative

$\mathrm{n}=$ Total number of crocetin derivative

\subsubsection{The crocetin derivative fraction identification by MS}

The MS characteristics of each crocetin derivative fraction were further acquired by the online MS detector under the following conditions: Spray voltage $=-4.0 \mathrm{Kv}$; Sheath gas $=30 \mathrm{arb}$; Auxiliary gas $=10 \mathrm{arb}$; Purge gas $=0$ arb; Capillary temperature $=350{ }^{\circ} \mathrm{C}$; Negative ion detection mode; The secondary mass spectra data acquisition: Data dependency scan; CID collision energy $=35 \%$; Fragment mass scanning range $=150-2000(\mathrm{~m} / \mathrm{z})$. The molecular ion peak was for each crocetin derivate was carefully identified and verified. The $\mathrm{m} / \mathrm{z}$ value of each molecular ion was recorded.

The crocetin derivative fractions were identified by MS and the secondary MS according to the presence of crocetin ion $=327[\mathrm{~m} / \mathrm{z}]$, as the root structure of the molecules.

1.3.5 The specific absorbance coefficient measurement of each crocetin derivative

The specific absorption coefficient of each crocetin derivative fraction is calculated from the published absorption coefficient, $\mathrm{A}_{1 \mathrm{~cm}}^{1 \%}=3820$ (in $\mathrm{EtOH}$ ), of crocetin as root structure, in accordance with the negative dependence of crocetin derivative UV-VIS absorbance on its side-chain quantity and mass indicated by molecular mass as shown in formula (3). The molecular mass of each crocetin derivative is hereon substituted by its measured parent ion [M-1] $]^{-}$mass reading $[\mathrm{m} / \mathrm{z}]$ from MS.

$$
A_{1 m_{i}}^{1 \%}=A_{1 m_{\text {crocetin }}^{1 \%}} \times \frac{\text { Molecular mass } \text { crocetin }_{\text {Molecular mass }}}{\text { Mol }}
$$

Where

$A_{1 \mathrm{~cm}_{\mathrm{i}}}^{1 \%}=$ Absorbance coefficient of crocetin derivative $\mathrm{i}$, defined as the theoretical absorbance of a $1 \%(\mathrm{~W} / \mathrm{V})$ crocetin derivative $\mathrm{i}$ solution in a cell of $1 \mathrm{~cm}$ path-length at monitored $440 \mathrm{~nm}$.

$A_{1 \mathrm{~cm}_{\text {Crocetin }}^{1 \%}}=$ Absorbance coefficient of crocetin, defined as the theoretical absorbance of a $1 \%(\mathrm{~W} / \mathrm{V})$ crocetin solution in a cell of $1 \mathrm{~cm}$ path-length monitored at $440 \mathrm{~nm}$. Published value of 3820 (in methanol) is applied hereon.

Molecular mass crocetin= Molecular mass of crocetin. Published value of 328 is applied hereon.
Molecular mass ${ }_{\mathrm{I}}=$ Molecular mass of crocetin derivative i. Molecular mass of each crocetin derivative is hereon substituted by its parent ion mass $[\mathrm{m} / \mathrm{z}]$ from MS.

$\mathrm{i}=$ Fraction number of crocetin derivative

1.3.6 The specific absorbance coefficient measurement of total crocetin derivative

The specific absorption coefficient of total crocetin derivative is calculated by weighted sum from the relative peak area and absorption coefficients of each crocetin derivative as the results of formulae (2) and (3), respectively, according to formula (4).

$$
A_{1 \mathrm{~cm}}^{1 \%} m_{\text {Total crocetin derivstive }}=\sum_{i=1}^{n} \text { Relatvie peak area }{ }_{i} \times A_{1 \mathrm{~cm}}^{1 \%}
$$

Where

Relative peak area $\mathrm{i}=$ Relative peak area of crocetin derivative $\mathrm{i}$

$\mathrm{A}_{1 \mathrm{~cm}}^{1 \%}$ Total crocetin derivative $=$ Absorption coefficient of total crocetin derivative, defined as the theoretical absorbance of a $1 \%(\mathrm{~W} / \mathrm{V})$ total crocetin derivative solution in a cell of $1 \mathrm{~cm}$ path-length monitored at $440 \mathrm{~nm}$.

$A_{1 \mathrm{~cm}_{\mathrm{i}}}^{1 \%}=$ Absorbance coefficient of crocetin derivative $\mathrm{i}$, defined as the theoretical absorbance of a $1 \%(\mathrm{~W} / \mathrm{V})$ crocetin derivative i solution in a cell of $1 \mathrm{~cm}$ path-length monitored at $440 \mathrm{~nm}$.

$\mathrm{i}=$ Fraction number of crocetin derivative

$\mathrm{n}=$ Total number of crocetin derivative

\subsubsection{The relative content measurement of total crocetin derivative}

The relative content of total crocetin derivative in total pigment is calculated from the peak area of each fraction monitored at $440 \mathrm{~nm}$ according to formula (5).

$$
\text { Relative content } \text { Total crocetin derivative }=\frac{\sum_{i=1}^{n} \text { Peak area }_{i}}{\sum_{j=1}^{m} \text { Peak area }_{j}}
$$

Where

Relative content Total crocetin derivative $=$ Relative content of total crocetin derivative in total pigment

Peak area $\mathrm{i}=$ Peak area of crocetin derivative $\mathrm{i}$

Peak area ${ }_{j}=$ Peak area of fraction $j$

$\mathrm{i}=$ Fraction number of crocetin derivative

$\mathrm{n}=$ Total number of crocetin derivative

$\mathrm{j}=$ Fraction number

$\mathrm{m}=$ Total fraction number

\subsubsection{Measurement of the total crocetin derivative amount}

According to Lambert-Beer law, the amount of total crocetin derivative in gardenia yellow sample is calculated from the absorbance of the gardenia yellow aqueous solution, the relative content of total crocetin derivative in total pigment, and the specific absorption coefficient of total crocetin derivative as shown by formula (6).

$$
\begin{aligned}
& \text { Amount }_{\text {Total crocetin derivative }}[g]= \\
& \underline{A \times \text { Relative content }_{\text {Total crocetin derivative }} \times V \times N} \\
& A_{1 c m_{\text {Total crocetin derivative }}^{1 \%}} \times 100
\end{aligned}
$$

Where

Amount Total crocetin derivative $[\mathrm{g}]=$ Amount of total crocetin derivative in the gardenia yellow sample 
$\mathrm{A}=$ Absorbance of sample solution

$\mathrm{V}=$ Solvent volume for sample originally dissolving

$\mathrm{N}=$ Dilution fold of sample solution

Relative content Total crocetin derivative $=$ Relative content of total crocetin derivative in total pigment

$\mathrm{A}_{1 \mathrm{~cm}}^{1 \%}$ Total crocetin derivative $=$ Absorption coefficient of the total crocetin derivative, defined as the theoretical absorbance of a $1 \%(\mathrm{~W} / \mathrm{V})$ total crocetin derivative solution in a cell of $1 \mathrm{~cm}$ path-length monitored at $440 \mathrm{~nm}$.

1.3.9 The relative content measurement of total crocetin derivative

The relative content of total crocetin derivative in the gardenia yellow sample is calculated from the amount of total crocetin derivative and sample weight according to formula (7).

\section{Relative content ${ }_{\text {Total crocetin derivative }}[\%]=$ $\frac{\text { Amount }_{\text {Total crocetin derivative }}}{M} \times 100 \%$

Where

Content Total crocetin derivative $[\%]=$ Content of total crocetin derivative in the gardenia yellow sample

Amount Total crocetin derivative $[\mathrm{g}]=$ Amount of total crocetin derivative in the gardenia yellow sample

$\mathrm{M}=$ Mass of the gardenia yellow sample

\subsubsection{The relative content measurement of each crocetin derivative}

According to Lambert-Beer law, the relative content of each crocetin derivative in total crocetin derivative is calculated from the peak area and absorption coefficients of each crocetin derivative as shown by formula (8).

$$
\text { Relative content }_{i}[\%]=\frac{\frac{\text { Peak area }_{i}}{A_{11 \% m_{i}}^{1 \%}}}{\sum_{i=1}^{n} \frac{\text { Peak area }_{i}}{A_{1 c m_{i}}^{10 /}}} \times 100 \%
$$

Where

Relative content $\mathrm{i}=$ Relative content of each crocetin derivative in total crocetin derivative

Peak area $\mathrm{i}=$ Peak area of crocetin derivative $\mathrm{i}$

$i=$ Fraction number of crocetin derivative

$\mathrm{n}=$ Total number of crocetin derivative

1.3.11 Measuring the amount of each crocetin derivative

The amount of each crocetin derivative from the gardenia yellow sample is calculated from the relative content of each crocetin derivative and the total amount of crocetin derivative according to formula (9).

$$
\begin{gathered}
\text { Amount }_{i}[g]=\text { Relative content }_{i} \times \\
\text { Amount }_{\text {Total crocetin derivative }}
\end{gathered}
$$

Where

Amount $\mathrm{i}=$ Amount of each crocetin derivative from the gardenia yellow sample

Relative content $\mathrm{i}=$ Relative content of each crocetin derivative in total crocetin derivative

Amount Total crocetin derivative $=$ Amount of total crocetin derivative in the gardenia yellow sample $i=$ Fraction number of crocetin derivative

1.3.12 The relative crocetin content measurement of each crocetin derivative

According to formula (10), the relative crocetin content of each crocetin derivative is calculated from the molecular masses of crocetin and its derivative, 328Da and the parent ion mass of the derivative respectively.

$$
\begin{gathered}
\text { Relative crocetin content }{ }_{i}=\frac{\text { Crocetin molecular mass }}{\text { Crocetin derivative molecular mass }}+ \\
100 \% \quad \text { (10) }
\end{gathered}
$$

Where

Relative crocetin content $\mathrm{i}=$ Relative crocetin content of each crocetin derivative

Crocetin molecular mass $=328 \mathrm{Da}$

Crocetin derivative molecular mass $\mathrm{i}$ : The molecular mass of crocetin derivative $\mathrm{i}$ is hereon substituted by its parent ion [M-1] mass value $[\mathrm{m} / \mathrm{z}]$ from $\mathrm{MS}$.

$\mathrm{i}=$ Fraction number of crocetin derivative

1.3.13 The relative crocetin content measurement from the total crocetin derivative

The relative crocetin content of total crocetin derivative is calculated from the relative crocetin content of each crocetin derivative and its relative peak area by weighted sum.

$$
\begin{aligned}
& \text { Relative crocetin content } \\
& \sum_{i=1}^{n} \text { Retal crocetin derivative }_{\text {Re }}=
\end{aligned}
$$

Where

Relative crocetin content Total crocetin derivative $=$ Relative crocetin content of total crocetin derivative

Relative crocetin content $\mathrm{i}=$ Relative crocetin content of each crocetin derivative

Peak area $\mathrm{i}=$ Peak area of crocetin derivative $\mathrm{i}$

$\mathrm{i}=$ Fraction number of crocetin derivative

1.3.14 The total crocetin amount measurement of gardenia yellow sample

The total crocetin amount of gardenia yellow sample is calculated from the relative crocetin content of total crocetin derivative and the amount of total crocetin derivative.

$$
\begin{aligned}
& \text { Total crocetin amount }[g]= \\
& \text { Relative crocetin content } \text { Total crocetin derivative } \times \\
& \text { Amount } \text { Total crocetin derivative }_{\text {(12) }}
\end{aligned}
$$

Where

Relative crocetin content Total crocetin derivative $=$ Relative crocetin content of total crocetin derivative

Amount Total crocetin derivative $=$ Amount of total crocetin derivative in the gardenia yellow sample

1.3.15 Recovery, reproducibility and minimum detection limit

The amount equal to the crocin amount from gardenia yellow product adduced from external references was added to the sample before the analysis for the recovery, reproducibility and RSD assessment of the method. Additionally, minimum detection limit is also detected with added crocin. 
The final result is calculated from the arithmetic mean of parallel measurements. The absolute difference of two independent observations is not more than $5 \%$ of their arithmetic mean under the same conditions.

\section{Results}

\subsection{The specific absorption coefficient determination of} gardenia yellow by UV-VIS

The absorbance (A) of the diluted gardenia yellow aqueous solution at the concentration of $10.04 \mu \mathrm{g} / \mathrm{mL}$ was 0.5064 at $440 \mathrm{~nm}$. The specific

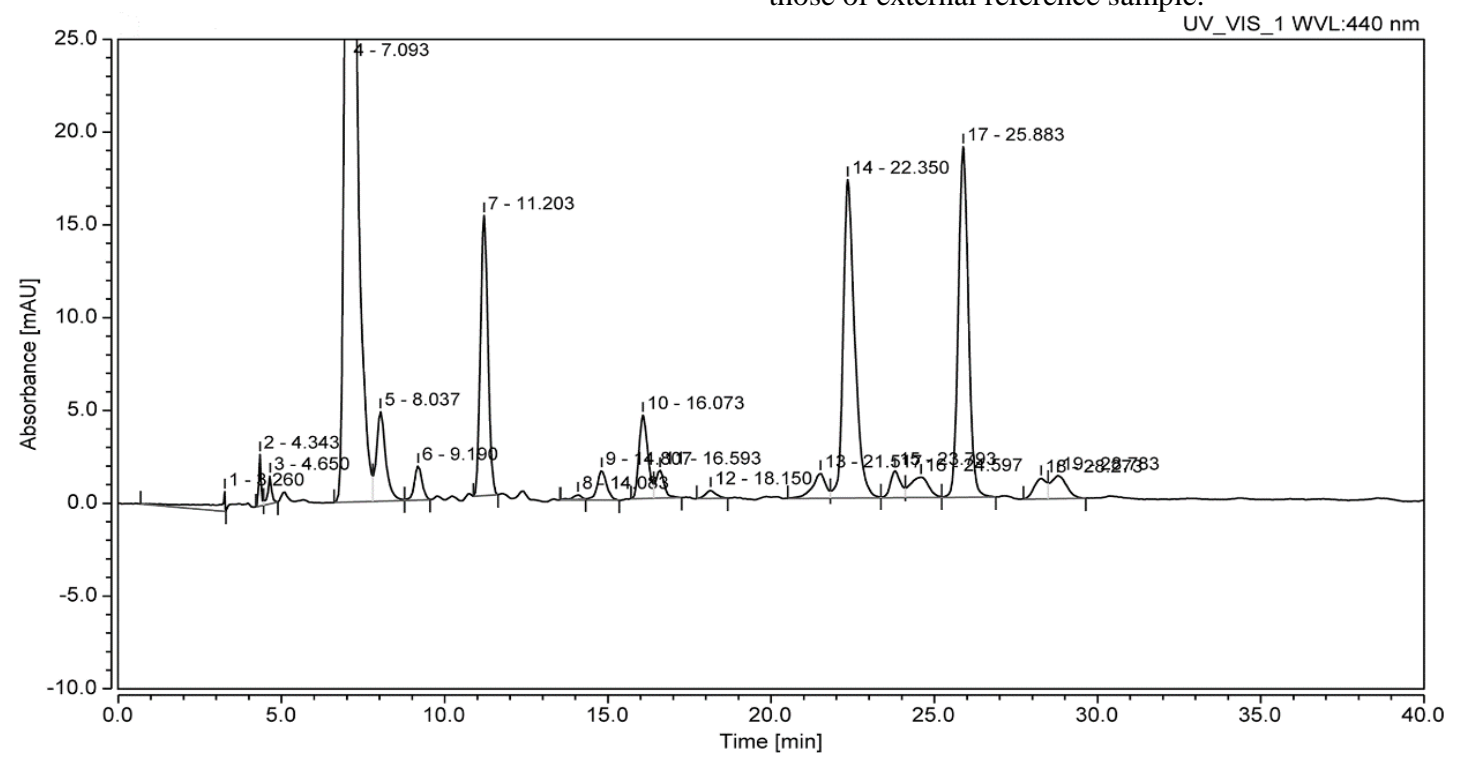
calculated according to formula (1).

\subsection{The C18-HPLC profile of gardenia yellow} those of external reference sample.

UV VIS 1 WVL: $440 \mathrm{~nm}$

absorption coefficient at $440 \mathrm{~nm}\left(E_{1 \mathrm{~cm}}^{1 \%}\right)$ of gardenia yellow was 504.38 ,

Figure 2 illustrates the C18-HPLC profile of gardenia yellow. It was observed from Figure 2 that the crocetin and its derivatives were well separated under the conditions employed in this study. The crocin fraction was identified in comparison of its retention time $=7.093 \mathrm{~min}$, its $\lambda_{\max }=$ $440 \mathrm{~nm}$ and the fine structure of its electronic absorption spectrum with

\section{Figure 2. The C18-HPLC profile of gardenia yellow. HPLC conditions: as shown in Section 1.3.2.}

\subsection{The fraction identification of gardenia yellow by HPLC-MS-MS}

Through MS and the secondary MS, the crocetin derivative fractions were carefully identified as the root structure of the molecules from the presence of crocetin ion $=327[\mathrm{~m} / \mathrm{z}]$. Under the conditions of ionization employed in this study, the major fragmentation taken placed during the MS process was the breaking of glycoside bonds. As an example, Figure 3 gives the TIC (a) of gardenia yellow and the mass spectrum (b) and secondary mass spectrum (c) of fraction at $7.093 \mathrm{~min}$. The ions

$$
\text { RT: } 0.00-40.00
$$
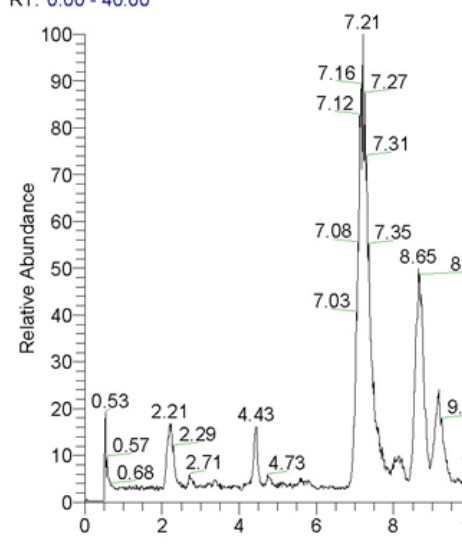

1089.34(100), 975.35 and 651.25 were seen from Figure 3(b). The ion 1089.34 was identified as parent ion [M-1]-. The presence of ions 975.35 and 651.25 suggested that the parent ion consisted of gentibiosidecrocetin-gentibioside as the root structure. This was further verified by the presence of ions 1089.34(100), 975.35, 651.25 and 327.15 from the secondary spectrum in Figure 3(c). The fraction would therefore be considered as a crocetin derivative.

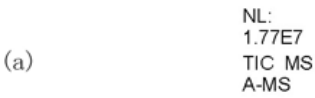


A-MS \#439 RT: 7.11 AV: 1 NL: $1.31 E 6$

T: FTMS - c ESI Full ms [100.00-2000.00]

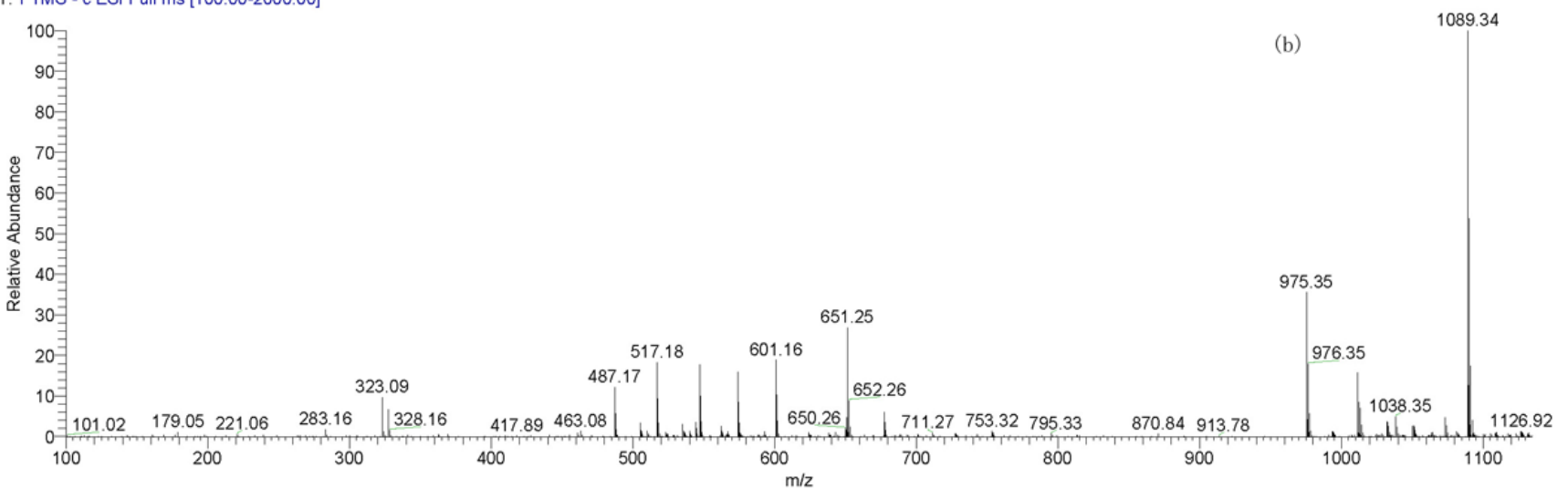

(b)

A-MS2 \#877 RT: 7.11 AV: 1 NL: $1.99 E 6$

T: FTMS - c ESI Full ms [100.00-2000.00]

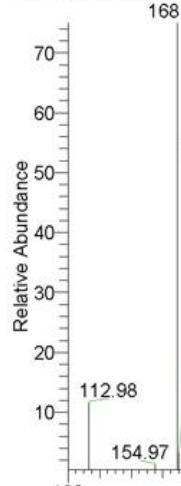
168.99

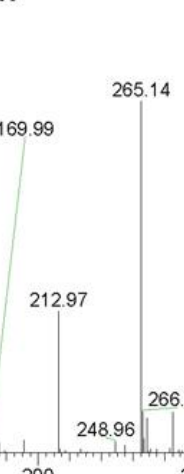

200

(c)

100

300

300

c)

Figure 3. MS characteristics of fraction at $7.093 \mathrm{~min}$ from gardenia yellow. (a)The TIC of gardenia yellow. (b)The mass spectrum of fraction at

$7.093 \mathrm{~min}$. (c) The secondary mass spectrum of fraction at 7.093 min. HPLC-MS-MS conditions: as shown in Sections 1.3.2 and 1.3.4.

2.4 The relative peak area of each fraction on the HPLC chromatogram of gardenia yellow

It is observed from Figure 2 that each fraction of gardenia yellow sample was readily separated under HPLC conditions as mentioned above. The peak area of each fraction was integrated. The relative peak area of each fraction was then calculated and given in Table 1.

\begin{tabular}{|c|c|c|c|c|c|c|c|c|c|}
\hline $\begin{array}{l}\text { Fracti } \\
\text { on No. }\end{array}$ & $\begin{array}{c}\text { Retention } \\
\text { time } \\
\text { [min] }\end{array}$ & $\begin{array}{c}\text { Peak area } \\
{\left[\mathrm{mAU}^{*} \text { min }\right.} \\
]\end{array}$ & $\begin{array}{c}\text { Relative } \\
\text { peak area } \\
{[\%]}\end{array}$ & $\begin{array}{c}\text { Parent ion } \\
{[\mathrm{m} / \mathrm{z}]}\end{array}$ & $\begin{array}{c}\text { Crocetin } \\
\text { piece } \\
{[\mathrm{m} / \mathrm{z}]}\end{array}$ & $\begin{array}{c}\mathrm{A}_{1 \mathrm{~cm}}^{1 \%} \text { of } \\
\text { crocetin } \\
\text { derivative } \mathrm{i}\end{array}$ & $\begin{array}{l}\text { Relative } \\
\text { content of } \\
\text { crocetin } \\
\text { derivative } \\
\text { i\%] }\end{array}$ & $\begin{array}{c}\text { Amount } \\
\text { of crocetin } \\
\text { derivative } \\
\text { i[g] }\end{array}$ & $\begin{array}{l}\text { Relative } \\
\text { crocetin } \\
\text { content of } \\
\text { crocetin } \\
\text { derivative } \\
\text { i [\%] }\end{array}$ \\
\hline 1 & 3.260 & 0.464 & 0.88 & - & - & - & - & - & - \\
\hline 2 & 4.343 & 0.201 & 0.38 & - & - & - & - & - & - \\
\hline 4 & 7.093 & 26.386 & 49.92 & 1089 & 327 & 1151 & 51.19 & 0.0115 & 30.02 \\
\hline 5 & 8.037 & 1.617 & 3.06 & 1089 & 327 & 1151 & 3.14 & 0.0007 & 30.02 \\
\hline 6 & 9.190 & 0.507 & 0.96 & 1089 & 327 & 1151 & 0.98 & 0.0002 & 30.02 \\
\hline 7 & 11.203 & 4.000 & 7.57 & 1137 & 327,651 & 1102 & 8.10 & 0.0018 & 28.75 \\
\hline 8 & 14.083 & 0.075 & 0.14 & 1241 & 327 & 1010 & 0.17 & 0.0000 & 26.34 \\
\hline 12 & 18.150 & 0.165 & 0.31 & - & - & - & - & - & - \\
\hline 13 & 21.517 & 0.638 & 1.21 & 819 & 327 & 1530 & 0.93 & 0.0002 & 39.92 \\
\hline
\end{tabular}




\begin{tabular}{|c|c|c|c|c|c|c|c|c|c|}
\hline 14 & 22.350 & 7.005 & 13.25 & 1241 & 327 & 1010 & 15.49 & 0.0035 & 26.34 \\
\hline 15 & 23.793 & 0.527 & 1.00 & 963 & 327 & 1301 & 0.90 & 0.0002 & 33.95 \\
\hline 16 & 24.597 & 0.721 & 1.36 & 1241 & 327 & 1010 & 1.59 & 0.0004 & 26.34 \\
\hline 17 & 25.883 & 6.743 & 12.76 & 951 & 327 & 1318 & 11.42 & 0.0026 & 34.38 \\
\hline 18 & 28.273 & 0.477 & 0.90 & 813 & 327,651 & 1541 & 0.69 & 0.0002 & 40.22 \\
\hline 19 & 28.783 & 0.694 & 1.31 & 813 & 327,651 & 1541 & 1.01 & 0.0002 & 40.22 \\
\hline Sum & & 52.854 & 100.0 & & & & 100.0 & 0.0225 & 29.35 \\
\hline
\end{tabular}

Table 1. The chromatographic behaviors, MS characteristics and amounts of each crocetin derivative fraction from gardenia yellow.

\subsection{Crocetin derivative fraction identification by MS}

The crocetin derivative fractions were identified by MS as the root structure of the molecules according to the presence of crocetin ion $=327$ $\mathrm{m} / \mathrm{z}$. The fractions containing crocetin structure are given in Table 1 . The parent ion $\mathrm{m} / \mathrm{z}$ value of each crocetin derivative is also recorded in Table 1 .

\subsection{The specific absorbance coefficient of each crocetin derivative}

With reference to the published specific absorption coefficient of crocetin, the specific absorption coefficient of each crocetin derivative fraction is calculated in accordance with the negative dependence of crocetin derivative UV-VIS absorbance on its side-chain quantity and mass indicated by molecular mass given in Table 1 .

\subsection{The specific absorbance coefficient of total crocetin derivative}

The specific absorption coefficient of the total crocetin derivative was calculated by the weighted sum from the relative peak area and the specific absorption coefficients of each crocetin derivative to be 1125 .

\subsection{The relative content of total crocetin derivative}

The relative content of total crocetin derivative in total pigment is calculated to be $97.20 \%$, from the peak area of each fraction monitored at $440 \mathrm{~nm}$.

\subsection{Total crocetin derivative amount}

The amount of total crocetin derivative in gardenia yellow sample is calculated to be $0.0225 \mathrm{~g}$ from the absorbance of gardenia yellow aqueous solution (measured by UV-VIS), the relative content of total crocetin derivative in total pigment and the specific absorption coefficient of total crocetin derivative.

\subsection{The relative content of total crocetin derivative from gardenia yellow}

The relative content of total crocetin derivative in the gardenia yellow sample is calculated to be $44.82 \%$ (W/W) from the amount of total crocetin derivative and sample weight.

\subsection{The relative content of each crocetin derivative}

According to Lambert-Beer law, the relative content of each crocetin derivative in total crocetin derivative is calculated from the peak area and absorption coefficients of each crocetin derivative and illustrated in Table 1 .

\subsection{The amount of each crocetin derivative}

The amount of each crocetin derivative from the gardenia yellow sample is calculated from the relative content of each crocetin derivative and the amount of total crocetin derivative and is given in Table 1.

\subsection{The relative crocetin content of each crocetin derivative}

The relative crocetin content of each crocetin derivative is calculated from the molecular masses of crocetin and its derivative, 328Da and the parent ion mass of the derivative respectively. The relative crocetin content of each crocetin derivative is given in Table 1.

\subsection{The relative crocetin content of total crocetin derivative}

The relative crocetin content of total crocetin derivative is calculated to be $29.35 \%$ from the relative crocetin content of each crocetin derivative and its relative peak area by weighted sum.

\subsection{The total crocetin amount of gardenia yellow sample}

From the relative crocetin content of total crocetin derivative and the amount of total crocetin derivative, the total crocetin amount of gardenia yellow is calculated to be $0.0066 \mathrm{~g}$ in the $0.0502 \mathrm{~g}$ gardenia yellow sample. The relative crocetin amount of gardenia yellow is $13.15 \%$ (W/W).

\subsection{Precision and accuracy}

To evaluate the precision and accuracy of the method as described above, $0.0115 \mathrm{~g}$ external reference sample of crocin (equal to the amount of crocin derivative fraction at $7.093 \mathrm{~min}$ from gardenia yellow sample) was added to the $0.0502 \mathrm{~g}$ gardenia yellow sample. The recovery, reproducibility and relative standard deviation (RSD) of added external reference sample were consequently assessed. Additionally, minimum detection limit was also detected.

It was observed that the RSD of repeated assessments for more than 6 times was less than $3.17 \%$. This observation suggested that the method exhibited good reproducibility. Additionally, the recovery of added crocin was more than $97 \pm 1.61 \%$. The minimum detection limit of crocin was $7.44 \mu \mathrm{g} / \mathrm{mL}$.

\section{Conclusion}

The major color materials from gardenia yellow are a group of crocetin derivatives. The molecular structures of some derivatives are well elucidated while those of the others are unknown and their commercial refence samples are short. The total amount and composition determinations of crocetin derivative are therefore currently difficult. The method developed in this study provides a possibility to assess each of the total derivative amounts through their absorption coefficients, although the molecular structures of some derivatives are unknown.

\section{References}

1. Yang, B., Liu, X and Gao, Y. (2009) Extraction optimization of bioactive compounds (crocin, geniposide and total phenolic compounds) from Gardenia (Gardenia jasminoides Ellis) fruits with response surface methodology. Innovative Food Science and Emerging Technologies, 10(4): 610-615.

2. Wang, X., Dong, S and Chen, F. (2012) Ultrasonic-assisted extraction of gardenia yellow from fructus Gardeniae. Advanced Materials Research, 396-398.

3. Zhu, X., Mang, Y., Shen, F., Xie, J., Su, W. (2014). Homogenate 
extraction of gardenia yellow pigment from Gardenia Jasminoides Ellis fruit using response surface methodology. Journal of Food Science and Technology, (51)8: 1575-1581.

4. Chen, J. F., Fu, G. M., Wan, Y., Liu, C. M., Chai, J. X., Li, H. G., Wang, J. T., Ming-Hu, Zhang, L. N. (2012). Enrichment and purification of gardenia yellow from Gardenia jasminoides var. radicans Makino by column chromatography technique Journal of Chromatography B, 893-894.

5. Feng, J., He, X., Zhou, S., Peng, F., Liu, J., Hao, L., Li, H., Ao, G., Yang, S. (2014). Preparative separation of crocins and geniposide simultaneously from gardenia fruits using macroporous resin and reversed-phase chromatography. Journal of Separation Science, 37(3): 314-322.

6. Choi, H. J., Park, Y.S., Kim, M.G., Kim, T.K., Yoon, N.S., Lim, Y.J. (2001). Isolation and characterization of the major colorant in Gardenia fruit. Dyes and Pigments, 49(1): 15-20.

7. Pfander H, Wittwer F. Carotenoid-glycosides. (1975). Investigation of carotenoid-composition of saffron. Helvetica chimica acta, 58 (6): 1608-1620.

8. Tarantilis P.A, Polissiou M.G, Manfait M. (1994). Separation of picrocrocin, cis-trans-crocins and safranal of saffron using highperformance liquid chromatography with photodiode-arraydetection. J. Chromatogr., A, 664: 55-61.

9. Tarantilis P.A, Tsoupras G, Polissiou M.G. (1995). Determination of saffron (Crocus sativus L.) components in crude plant extract using high-performance liquid chromatography-UV-visible photodiode-array detection-mass spectrometry. J. Chromatogr., A, 699(1): 107-118.

10. Lech K, Witowska-Jarosz J, Jarosz M. (2009). Saffron yellow: characterization of carotenoids by high performance liquid chromatography with electrospray mass spectrometric detection. Journal of Mass Spectrometry, 44(12): 1661-1667.

11. Pfister S, Meyer P, Steck A, Pfander H. (1996). Isolation and structure elucidation of carotenoid-glycosyl esters in gardenia fruits (Gardenia jasminoides Ellis) and saffron (Crocus sativus Linne). Journal of agricultural and food chemistry, 44(9): 26122615.

12. Van Calsteren M.R, Bissonnette M.C, Cormier F, Dufresne Ch, Ichi T, Yves Le Blanc J.C, Perreault D, Roewer I. (1997). Spectroscopic characterization of crocetin derivates from Crocus sativus L. and Gardenia jasminoides. J. Agric. Food Chem, 45: 1055-1061.

13. Straubinger M, Jezussek M, Waibel R, Winterhalter P. (1997). Novel glycosidic constituents from saffron. J. Agric. Food Chem., 45:1678-1681.

14. Carmona M, Zalacain A, Sánchez A.M, Novella J.L, Alonso G.L. (2006). Crocetin esters, picrocrocin and its related compounds present in Crocus sativus stigmas and Gardenia jasminoides fruits. Tentative identification of seven new compounds by LC-ESI-MS. Journal of agricultural and food chemistry, 54(3): 973-979.

15. Uekusa, Y., Sugimoto, N., Sato, K., Yun, Y.S., Kunugi, A., Yamazaki, T., Tanamoto, K. I. Neocrocin A. (2007). A novel crocetin glycoside with a unique system for binding sugars isolated from gardenia yellow. Chemical and Pharmaceutical Bulletin, 55(11): 1643-1646.

16. He W, Liu X, Xu H, Gong Y, Yuan F, Gao Y. (2010). On-line HPLC-ABTS screening and HPLC-DAD-MS/MS identification of free radical scavengers in Gardenia fruit extracts. Food Chemistry, 123(2): 521-528.

17. Park, Y.S., Choi, H.J., Yun, S.H., Kim, S.H., Kim, T.K., Yoon, N.S., Lim, Y.J. (2001). Quantitative analysis of crocetin colorants in gardenias (Gardenia jasminoides Ellis) by LC/DAD/MS Journal of Industrial and Engineering Chemistry, 7(6): 375-379.

18. Zhou, W. E., Zhang, Y., Li, Y., Ling, Y., Li, H. N., Li, S. H., Jiang, S. J., Ren, Z. Q., Huang, Z. Q., Zhang, F. (2016). Determination of gardenia yellow colorants in soft drink, pastry, instant noodles with ultrasound-assisted extraction by high performance liquid chromatography-electrospray ionization tandem mass spectrum. Journal of Chromatography A, 1446: 59-69.

19. Yamada, S., Oshima, H., Saito, I., Hayakawa, J. (1996). Adoption of crocetin as an indicator compound for detection of gardenia yellow in food products (analysis of natural coloring matters in food. V) Journal of the Food Hygienic Society of Japan, 37(6): 372-377.

20. Inoue, K., Tanada, C., Nishikawa, H., Matsuda, S., Tada, A., Ito, Y., Min, J.Z., Todoroki, K., Sugimoto, N., Toyo'Oka, T., Akiyama, H. (2014). Evaluation of gardenia yellow using crocetin from alkaline hydrolysis based on ultra-high performance liquid chromatography and high-speed countercurrent chromatography. Journal of Separation Science, 37(24): 3619-3624.
This work is licensed under Creative Commons Attribution 4.0 License
Ready to submit your research? Choose Auctores and benefit from:

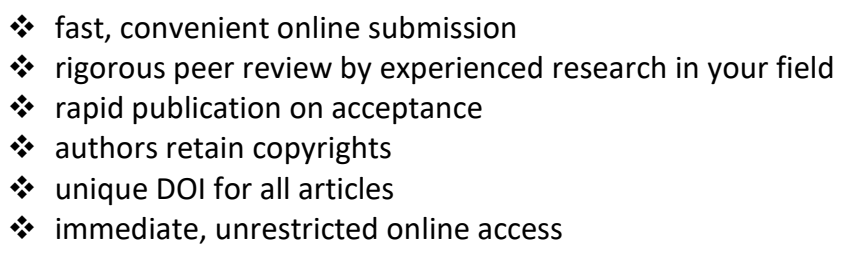

At Auctores, research is always in progress.

Learn more www.auctoresonline.org/journals/nutrition-and-foodprocessing 